Quantitative Political Economy Research Group

Department of Political Economy

King's College London

\title{
QPE
}

\section{Welfare Targeting in Networks}

QPE Working Paper 2020-15

Nizar Allouch

Maia King.

March 9, 2021 


\title{
Welfare targeting in networks*
}

\author{
Nizar Allouch ${ }^{\dagger}$ \\ University of Kent
}

\author{
Maia King $\ddagger$ \\ King's College London
}

March 9, 2021

\begin{abstract}
This paper investigates welfare targeting for public goods in networks. First, we show that a tax/subsidy scheme (not necessarily budget-balanced) affects each consumer only insofar as it affects his neighbourhood. Second, we show that either a Pareto-improving income redistribution can be found or there exist Negishi weights, which we relate to the network structure. Third, in the case of Cobb-Douglas preferences, we show that a law of welfare targeting holds and links two well-known notions of the comparative statics of policy interventions: neutrality and welfare paradoxical effects. Collectively, our findings uncover the importance of the -1 eigenvalue to economic and social policy: it is an indication of how consumers absorb the impact of income redistribution.
\end{abstract}

JEL classification: D85, H41.

Keywords: public goods, tax/subsidy, welfare, network, -1 eigenvalue.

${ }^{*}$ We would like to thank James Andreoni, Ted Bergstrom, Sebastian Bervoets, Larry Blume, Yann Bramoullé, Sergio Currarini, Matthew Elliott, Andrea Galeotti, Ben Golub, Sanjeev Goyal, Roger Guesnerie, Jaromír Kovárík, Miltos Makris, Hervé Moulin, and Junjie Zhou for valuable discussions, and one anonymous reviewer for their helpful comments and suggestions. For support, Nizar Allouch thanks ANR 18-CE26-0020-01. Declarations of interest: none.

${ }^{\dagger}$ School of Economics. E-mail: n.allouch@kent.ac.uk. Corresponding author.

${ }_{\ddagger}^{\ddagger}$ Department of Political Economy. E-mail: maia.king@kcl.ac.uk 


\section{Introduction}

Many major challenges facing modern societies (essential infrastructure, information acquisition, emerging infectious disease) relate to enhancing public good provision across different consumers. While market outcomes often provide scope for policy intervention, central planners seldom have the luxury to completely change the state of the economy and implement optimal outcomes. Instead, social planners typically aim to design welfareimproving reforms - involving small changes as in the policy reform literature in the tradition of Dixit (1975) and Guesnerie (1977), and with important policy implications as in Ahmad and Stern (1984).

This paper explores welfare targeting with small tax/subsidy schemes, not necessarily budget-balanced, which are traditionally viewed as a benchmark for broader policy interventions, for the private provision of public goods in networks. In a key contribution, Bramoullé and Kranton (2007) showed that the network context, where local influences are heterogeneous among consumers, is a natural setting to examine the private provision of public goods. Bramoullé, Kranton and D'Amours (2014) investigated the whole range of strategic substitution and identified a threshold of impact related to the lowest eigenvalue of the network. Below the threshold, the uniqueness and stability of a Nash equilibrium hold. Beyond it, multiple Nash equilibria will in general exist, and stability holds only for corner equilibria. Allouch (2015) extended this model to the non-linear case, with a condition on the normality of the public good which follows the classical Bergstrom, Blume and Varian (1986) (henceforth BBV) approach, and showed that their neutrality no longer holds for income redistribution in general networks. Galeotti, Golub and Goyal (2020) analyse optimal policy interventions informed by the eigenvalues of the underlying network of spillovers. Other recent and relevant contributions to the network literature include those by: Galeotti, Goyal, Jackson, Vega-Redondo and Yariv (2010); Ghiglino and Goyal (2010); Acemoglu, Malekian and Ozdaglar (2016); Bourlès, Bramoullé and Perez-Richet (2017); Kinateder and Merlino (2017); López-Pintado (2017); Chen, Zenou and Zhou (2018); Belhaj and Deroïan (2019); Elliott and Golub (2019); Allouch (2017); Allouch and King (2019); Akbarpour, Malladi and Saberi (2020); Ushchev and Zenou (2020); Li, Zhou and Chen (2021), and Sun, Zhao and Zhou (2021).

First, we establish a property that is key to understanding the impact of welfare targeting in networks. More specifically, we show that a tax/subsidy scheme affects each 
consumer only insofar as it affects the consumer's neighbourhood, formed by himself and his neighbours. That is, it is the neighbourhood scheme, rather than the tax/subsidy scheme that affects consumption. Hence the policy implications can be derived by focusing just on the neighbourhood schemes. In particular, neutral tax/subsidy schemes (budget-balanced or not) in general networks are those with null neighbourhood schemes, or equivalently those that are the eigenvectors to the -1 eigenvalue.

Secondly, to inform/guide welfare targeting, we use an approach based on a weighted utilitarian social welfare function. More specifically, the social planner maximises a weighted sum of consumers' utility functions. We find two mutually exclusive cases - either there is a Pareto-improving income redistribution or, if not, there exist Negishi welfare weights. These Negishi weights provide the implicit welfare weights at the initial equilibrium. In particular, we find that a Pareto-improving income redistribution always exists in the class of networks that has a neutral and budget-unbalanced tax/subsidy scheme, since Negishi weights cannot exist. This finding is very much in the spirit of the BBV neutrality result as it holds for any profile of preferences of consumers. Additionally, in the case of Cobb-Douglas preferences, we show that the feasibility of Pareto-improving reform turns out to be readily interpreted and easily checked from the network structure. As a consequence, our analysis leads to a useful characterisation of welfare targeting.

Thirdly, we provide a link between key, but seemingly unrelated, notions of comparative statics: neutrality and paradoxical welfare effects. In fact, by focusing on tax/subsidy schemes that are also eigenvectors, our analysis shows that in the case of Cobb-Douglas preferences, neutrality (or, equivalently, the -1 eigenvalue) corresponds to the point of policy switch between tax/subsidy schemes where the utility levels of the donors and the recipients move in the same direction as the scheme (normal welfare impact) and tax/subsidy schemes where the utility levels of the donors and the recipients move in the opposite direction (paradoxical welfare impact). In addition, we show that a law of welfare targeting holds for more general tax/subsidy schemes determined by the -1 eigenvalue.

In different settings, our results highlight the importance of the -1 eigenvalue to social and economic outcomes, since our findings identify it as a condition for neutral tax/subsidy schemes, Pareto improvement, and the policy switch. In interpretation, the -1 eigenvalue is an indication of how consumers, via their neighbourhood, absorb the impact of tax/subsidy schemes, and hence of the welfare implications. Despite the 
frequency with which the -1 eigenvalue appears for many (but not all) networks, as far as we know the -1 eigenvalue is not used as a common measure of network analysis in any other fields, including sociology, computer science, and physics. Given that the -1 eigenvalue provides a key to social and economic outcomes, perhaps its relationship to the underlying network structure could usefully be studied alongside classic network statistics such as the highest, the second, and lately the lowest eigenvalues.

The paper is structured as follows. Section 2 sets out the model and Section 3 investigates welfare targeting. Section 4 looks at Pareto improvement and Negishi weights and Section 5 provides a new perspective on neutrality and paradoxical welfare effects. Section 6 concludes the paper. An Appendix provides proofs of the propositions and corollaries.

\section{The model}

We consider a society comprising $n$ consumers in $N=\{1, \ldots, n\}$ who occupy the nodes of a fixed network $\mathbf{g}$ of social interactions. Let $\mathbf{G}=\left[g_{i j}\right]$ denote the adjacency matrix of the network $\mathbf{g}$, where $g_{i j}=1$ indicates that consumer $i \neq j$ are neighbours and $g_{i j}=0$ otherwise. The adjacency matrix of the network, $\mathbf{G}$, is symmetric with non-negative entries and therefore has a complete set of real eigenvalues (not necessarily distinct), denoted by $\lambda_{\max }(\mathbf{G})=\lambda_{1} \geq \lambda_{2} \geq \ldots \geq \lambda_{n}=\lambda_{\min }(\mathbf{G})$, where $\lambda_{\max }(\mathbf{G})$ is the largest eigenvalue and $\lambda_{\min }(\mathbf{G})$ is the lowest eigenvalue of $\mathbf{G}$. By the Perron-Frobenius Theorem, it holds that $\lambda_{\max }(\mathbf{G}) \geq-\lambda_{\min }(\mathbf{G})>0$.

Consumer $i$ 's neighbours in the network $\mathrm{g}$ are given by $\mathcal{N}_{i}$. The preferences of each consumer $i$ are represented by a twice continuously differentiable, strictly increasing, and strictly quasi-concave utility function $u_{i}\left(x_{i}, q_{i}+Q_{-i}\right)$, where $x_{i}$ is consumer $i$ 's private good consumption, $q_{i}$ is consumer $i$ 's public good provision, and $Q_{-i}=\sum_{j \in \mathcal{N}_{i}} q_{j}$ is the sum of public good provisions of consumer $i$ 's neighbours in the society. Furthermore, the public good can be produced from the private good via a unit-linear production technology. That is, any non-negative quantity of the private good can be converted into the same quantity of the public good. For simplicity, the prices of the private good and the public good can be normalised to $\mathbf{p}=\left(p_{x}, p_{Q}\right)=(1,1)$. Each consumer $i$ faces the 
utility maximisation problem

$$
\begin{array}{ll} 
& \max _{x_{i}, q_{i}} u_{i}\left(x_{i}, q_{i}+Q_{-i}\right) \\
\text { s.t. } & x_{i}+q_{i}=w_{i} \text { and } q_{i} \geq 0,
\end{array}
$$

where $w_{i}$ is his income (exogenously fixed). The utility maximisation problem can be represented equivalently as

$$
\begin{gathered}
\max _{x_{i}, Q_{i}} u_{i}\left(x_{i}, Q_{i}\right) \\
\text { s.t. } x_{i}+Q_{i}=w_{i}+Q_{-i} \text { and } Q_{i} \geq Q_{-i},
\end{gathered}
$$

where consumer $i$ chooses his (local) public good consumption, $Q_{i}=q_{i}+Q_{-i}$. Let $\gamma_{i}$ be the Engel curve of consumer $i$. Then consumer $i$ 's local public good demand depends on $w_{i}+Q_{-i}$, each consumer's 'social wealth as in Becker (1974):

$$
Q_{i}=\max \left\{\gamma_{i}\left(w_{i}+Q_{-i}\right), Q_{-i}\right\}
$$

or equivalently

$$
q_{i}=Q_{i}-Q_{-i}=\max \left\{\gamma_{i}\left(w_{i}+Q_{-i}\right)-Q_{-i}, 0\right\}
$$

We will assume, throughout the paper, the following network-specific normality assumption, which amounts to both the normality of the private good and a strong normality of the public good:

Definition 1. Network normality. Allouch (2015) For each consumer $i=1, \ldots, n$, the Engel curve $\gamma_{i}$ is differentiable and it holds that $1+\frac{1}{\lambda_{\min }(\mathbf{G})}<\gamma_{i}^{\prime}(\cdot)<1$.

Theorem 1. Allouch (2015) Assume network normality. Then there exists a unique Nash equilibrium in the private provision of public goods on networks.

\section{$3 \quad$ Welfare targeting}

In this paper, we investigate welfare targeting by a social planner in networks. The idea is that implementing a welfare-efficient outcome is typically unachievable because it requires a significant change from the existing state of the economy. More realistic are small tax/subsidy schemes, which induce infinitesimal changes that are welfare-improving 
and equilibrium-preserving as in Dixit (1975), Guesnerie (1977), and Ahmad and Stern (1984).

Let $\mathbf{q}^{*}=\left(q_{1}^{*}, \ldots, q_{n}^{*}\right)$ be the Nash equilibrium associated with $\mathbf{w}=\left(w_{1}, \ldots, w_{n}\right)$ and let $\mathbf{s}=\left(s_{1}, \ldots, s_{n}\right)$ denote a tax/subsidy scheme, where $s_{i}<0$ is a tax and $s_{i} \geq 0$ is a subsidy. Let $\mathbf{q}^{\mathbf{s}}=\left(q_{1}^{\mathbf{s}}, \ldots, q_{n}^{\mathbf{s}}\right)$ be the Nash equilibrium associated with $\mathbf{w}+\mathbf{s}=$ $\left(w_{1}+s_{1}, \ldots, w_{n}+s_{n}\right)^{T}$. Let also $\mathbf{v}^{*}=\left(u_{i}\left(x_{i}^{*}, Q_{i}^{*}\right)\right)_{i \in N}$ denote the vector of utilities of all consumers at the initial equilibrium, $\mathbf{v}^{\mathbf{s}}=\left(u_{i}\left(x_{i}^{\mathbf{s}}, Q_{i}^{\mathbf{s}}\right)\right)_{i \in N}$ denote the vector of utilities after a tax/subsidy scheme $\mathbf{s}$, and $\Delta \mathbf{v}(\mathbf{s})=\mathbf{v}^{\mathbf{s}}-\mathbf{v}^{*}$.

Typically, not all consumers will be contributing to public goods. Therefore, at a Nash equilibrium, there will be possibly several components of contributors. We will focus our analysis on just one component of contributors. For simplicity of notation, by passing to the subnetwork, we will assume $N$ is a component of contributors. Note that, by doing so, we can no longer consider the public goods consumption or welfare of non-contributors. Similarly, if we pass our analysis to several components of contributors, while we can fully characterise the provision of public goods and welfare of contributors, we can no longer consider the public good consumption or welfare of non-contributors.

Given a tax/subsidy scheme $\mathbf{s}$, let $\mathbf{S}=(\mathbf{I}+\mathbf{G}) \mathbf{s}$ denote the corresponding neighbourhood scheme. That is, the corresponding neighbourhood scheme $\mathbf{S}$ measures how the tax/subsidy scheme s affects each consumer's neighbourhood, formed by himself and his neighbours. In particular, we say $\mathbf{s}$ is neighbourhood balanced whenever $\mathbf{S}=\mathbf{0}$.

Proposition 1. Given a component of contributors, the change in consumers' utilities/welfare following a small tax/subsidy scheme $\mathbf{s}$ is

$$
\Delta \mathbf{v}(\mathbf{s}) \approx \mathbf{A}^{-1}(\mathbf{I}+\mathbf{A G})^{-1} \mathbf{A S}
$$

where $\mathbf{A}=\operatorname{diag}\left(1-\gamma_{i}^{\prime}\left(w_{i}+Q_{-i}^{*}\right)\right)_{i \in N}$.

Proposition 1 shows that it is the corresponding neighbourhood scheme, rather than the tax/subsidy scheme itself, that determines the welfare impact. That is, a tax/subsidy scheme impacts each consumer's welfare only insofar as it impacts his neighbourhood. As a consequence, policy implications can be derived from focusing just on the corresponding neighbourhood schemes. Note that a tax/subsidy scheme can yield a very different corresponding neighbourhood scheme, for some consumers not only in terms of magni- 
tude but also in terms of direction of impact. For instance, there can be consumers who are subsidized in the tax/subsidy scheme and taxed in the corresponding neighbourhood scheme, or conversely.

In particular, neutral tax/subsidy schemes, defined as those that are neighbourhood balanced, or equivalently, that have a null corresponding neighbourhood scheme, represent an important class. Neutral tax/subsidy schemes have no impact on consumers' welfare, and therefore reduce the scope of policy intervention.

Corollary 1. Given a component of contributors, small and neutral tax/subsidy schemes are the eigenvectors to the -1 eigenvalue.

Hence the eigenvectors to the -1 eigenvalue, not necessarily budget-balanced, characterise neutral tax/subsidy schemes for any profile of preferences. This provides a link between the network structure and the policy constraints faced by the policy maker.

There is a wealth of literature focusing on the neutrality of budget-balanced tax/subsidy schemes, or equivalently income redistribution. It is worth noting that the neutrality of BBV occurs as a limiting case, since only the complete network, which is equivalent to pure public goods, reaches the highest possible number $(n-1)$ of linearly independent eigenvectors to the -1 eigenvalue. Allouch (2015) shows that the neutrality of BBV extends to subsets of consumers that are similar in structure to pure public goods. In general, neutral tax/subsidy schemes can be either budget-balanced or budget-unbalanced and can also occur in network structures that are very dissimilar from pure public goods, as we illustrate in Figures 1 and $2 .^{1}$

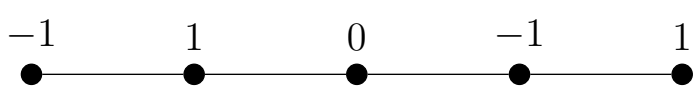

S

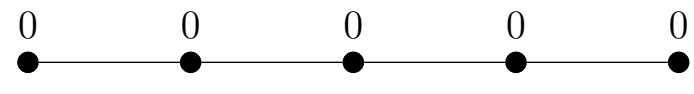

S

Figure 1: A neutral and budget-balanced tax/subsidy scheme in a line component of five contributors. On the left, $\mathbf{s}$ is the tax/subsidy scheme where some consumers are taxed and others are subsidised. On the right, $\mathbf{S}=(\mathbf{I}+\mathbf{G})$ s is the corresponding neighbourhood tax/subsidy scheme. Note that $\mathbf{S}=0$, which implies that $\mathbf{s}$ has no welfare impact, according to Proposition 1.

\footnotetext{
${ }^{1}$ We will provide an example of a neutral and budget-unbalanced tax/subsidy scheme in the next section (Figure 3).
} 


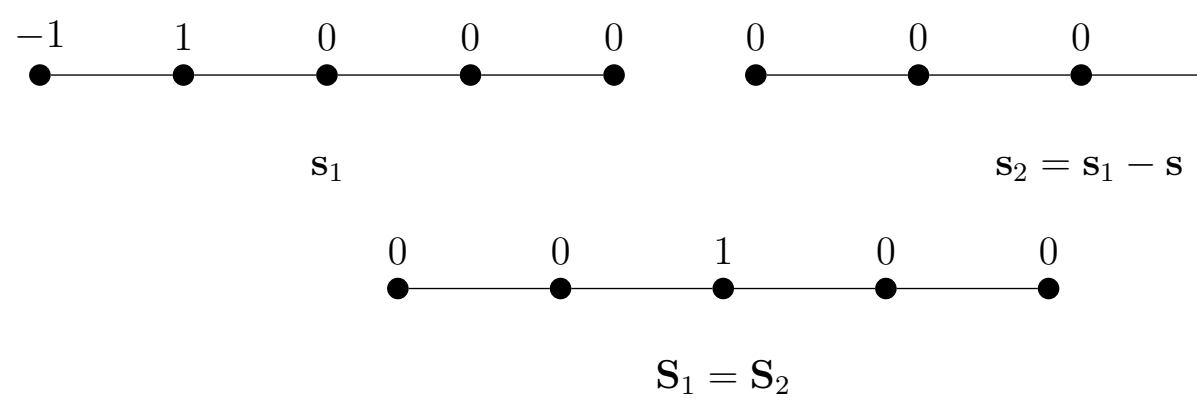

Figure 2: Policy-equivalent tax/subsidy schemes in the line component of five contributors. Two tax/subsidy schemes $\mathbf{s}_{1}$ and $\mathbf{s}_{2}$ have identical corresponding neighbourhood schemes $\mathbf{S}_{1}$ and $\mathbf{S}_{2}$. This means they are 'policy-equivalent' and would have the same welfare impact. It is worth noting that the two schemes $\mathbf{s}_{1}$ and $\mathbf{s}_{2}$ seem unrelated since the consumers involved with $\mathbf{s}_{1}$ are not adjacent to consumers involved with $\mathbf{s}_{2}$.

\section{Pareto improvement vs Negishi weights}

To inform welfare targeting, we follow an approach based on a weighted utilitarian social welfare function with welfare weights given by $\mathbf{r} \in \mathbb{R}_{++}^{n}$. Hence the social welfare effect of a tax/subsidy scheme $\mathbf{s}$ is $\mathbf{r} \cdot \Delta \mathbf{v}(\mathbf{s})$.

An interesting set of welfare weights are Negishi weights (named after Negishi (1960)), which neutralise any impact of policy change from income redistribution at the initial equilibrium, and are hence defined by $\mathbf{r} \cdot \Delta \mathbf{v}(\mathbf{s})=0$ for any budget-balanced tax/subsidy scheme s. Negishi weights are often used in climate change economics models to 'freeze' income distribution between countries (Stanton, 2011; Abbott and Fenichel, 2014) and, for example, to investigate magnitudes and directions of technological transfers (Yang and Nordhaus, 2006).

Proposition 2. Given a component of contributors, and where $\mathbf{R}=\mathbf{r}(\mathbf{I}+\mathbf{G})$, there exist Negishi weights if and only if there exists $\mathbf{r} \in \mathbb{R}_{++}^{n}$ such that

$$
\mathbf{R}=\mathbf{1}(\mathbf{I}-\mathbf{A})^{-1}(\mathbf{I}+\mathbf{A G}) .
$$

Proposition 2 relates the existence of Negishi weights to the network structure and the preferences of consumers. It characterises whether the Negishi weights, defined by $\mathbf{r} \cdot \Delta \mathbf{v}(\mathbf{s})=0$ for any budget-balanced tax/subsidy scheme, can be found in our setting. 
In particular, Proposition 2 shows that it is the neighbourhood Negishi weights $\mathbf{R}$ which matter for this existence result, which flows directly from the fundamental importance of the neighbourhood effects of any tax/subsidy scheme, shown in Proposition 1.

Since Negishi weights neutralise any policy impact from income redistribution, a key implication of their existence is that they rule out the possibility of a Pareto-improving income redistribution. In an analogous approach, Ahmad and Stern (1984) use the 'inverse optimum' to identify welfare weights that represent the implicit weights at the initial equilibrium. If these welfare weights do not exist, this implies that a Pareto improvement is possible. This method relates to an application of Motzkin's Theorem of the Alternative. The following result further links the existence of Negishi weights to Pareto improvement, in our setting of redistribution in networks with public goods.

Proposition 3. Given a component of contributors, there are only two (mutually exclusive) possibilities, (a) and (b):

(a) There exists a Pareto-improving income redistribution.

(b) There exist Negishi weights.

Proposition 3 shows that either a Pareto-improving income redistribution can be found or there exist Negishi weights. Hence, in every network structure, Pareto improvement is in conflict with the Negishi weights. Whenever they do exist, Negishi weights represent the implicit welfare weights at the initial equilibrium.

\subsection{Pareto improvement and the -1 eigenvalue}

Proposition 3 provides a simple method based on the network structure to check whether a Pareto-improving income redistribution can be found or not. In this regard, the link between the budget balance requirement and the vector 1 highlights the role of main eigenvalues in our analysis. ${ }^{2}$ A main eigenvalue is an eigenvalue that has an eigenvector not orthogonal to the vector 1 Cvetkovic (1970). ${ }^{3}$

\footnotetext{
${ }^{2}$ Quite differently, Allouch $(2015,2017)$ show that the expression of Bonacich centrality in terms of main eigenvalues has useful policy implications.

${ }^{3}$ By the Perron-Frobenius Theorem, the maximum eigenvalue of $\mathbf{G}$ has an associated eigenvector with all its entries positive and which, therefore, is a main eigenvalue.
} 
Proposition 4. Given a component of contributors, if -1 is a main eigenvalue then there exists a small Pareto-improving income redistribution.

Proposition 4 is very much in the spirit of the BBV neutrality since it holds for any profile of preferences. In interpretation, if -1 is a main eigenvalue, then a corresponding eigenvector is a neutral and budget-unbalanced tax/subsidy scheme. Therefore, Negishi weights cannot exist given that, in their presence, only budget-balanced tax/subsidy schemes are policy-neutral. On the other hand, the social planner can use the neutral and budget-unbalanced tax/subsidy scheme to create tax revenue which can be redistributed for Pareto improvement. More specifically, the social planner could either tax or subsidize consumers according to their entries in the neutral and budget-unbalanced tax/subsidy scheme and create a budget surplus, which can be redistributed for Pareto improvement. Figure 3 illustrates this.

In the following, we provide an example of network structures where -1 is a main eigenvalue.

Definition 2. A network is equal neighbourhood, if consumers can be partitioned into two unequal-size groups such that the number of links of each consumer to his group members, is equal to the number of his links to consumers in the other group minus one.

In interpretation, in an equal neighbourhood network each consumer has an equal neighbourhood size in each group. Given that the groups are unequal in size, but consumers have equal sized neighbourhoods in them, the tax/subsidy scheme where consumers in one group are uniformly taxed and consumers in the other group are uniformly subsidized is both neutral and budget-unbalanced, and hence is an eigenvector to the -1 eigenvalue. Therefore, -1 is a main eigenvalue. A corollary follows from this:

Corollary 2. Given an equal neighbourhood network, then -1 is a main eigenvalue. Hence, there exists a small Pareto-improving income redistribution.

An example of equal neighbourhood networks is a special class of core-periphery networks with $c$ fully connected core consumers, where each core consumer has $c$ periphery consumers connected only to her. ${ }^{4}$ This is illustrated in Figure 3.

\footnotetext{
${ }^{4}$ More generally, several classes of core-periphery networks feature frequently in economic analysis, including as the outcome of a public good network formation game by Galeotti and Goyal (2010).
} 


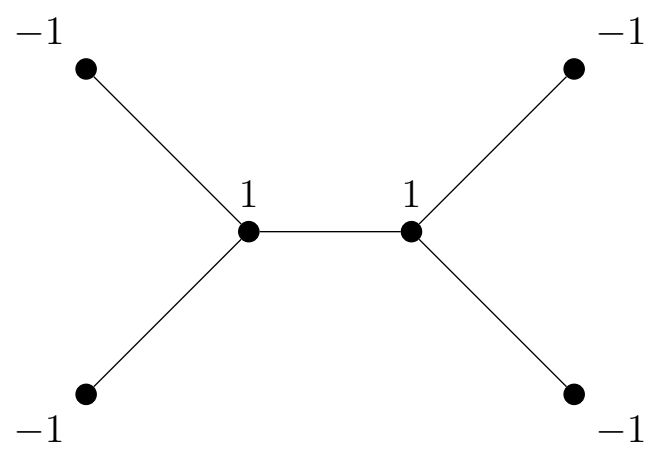

$\mathbf{S}$

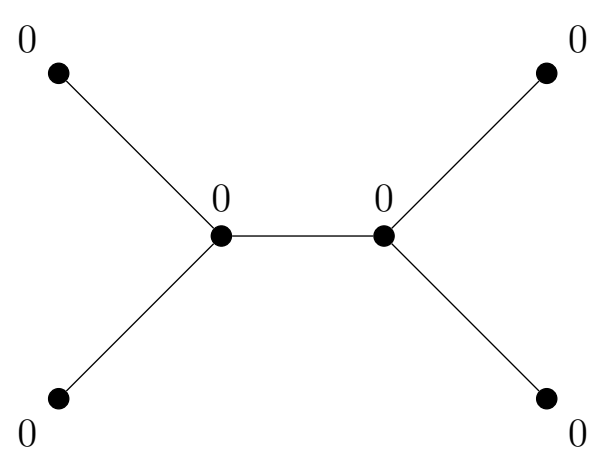

S

Figure 3: A neutral and budget-unbalanced tax/subsidy scheme in a core-periphery component of contributors, with two core consumers and four periphery consumers. We can observe that the tax/subsidy scheme $\mathbf{s}$ such that $s_{p}=-1$ for periphery consumers and $s_{c}=1$ for core consumers is budget-unbalanced but policy-neutral since its impact is null in each consumer's neighbourhood. Note that $\mathbf{s}$ is an eigenvector to the main eigenvalue -1 . Therefore, the social planner could use the tax/subsidy scheme $\mathbf{s}$ as follows: take 1 unit of income from each of the four periphery consumers, then subsidise the two core consumers with 1 unit of income each. This is policy-neutral and creates a budget surplus of 2 units of income which can be redistributed for Pareto improvement. 


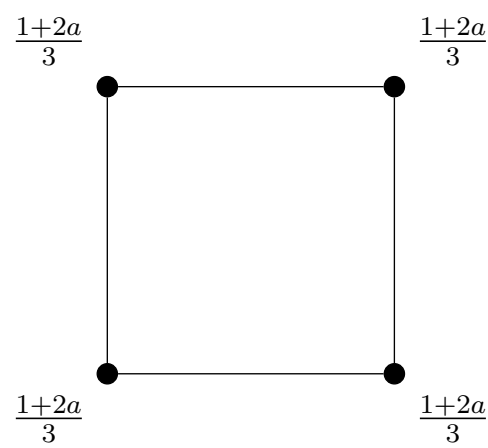

(a) A regular network

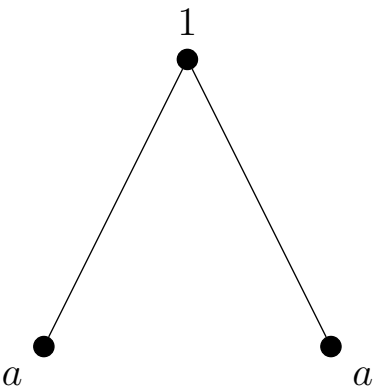

(b) A star network

Figure 4: Negishi weights in two networks. For the regular network with four consumers in Figure $4 \mathrm{a}$, the Negishi weights are $\mathbf{r}=\left(\frac{1+2 a}{3}, \frac{1+2 a}{3}, \frac{1+2 a}{3}, \frac{1+2 a}{3}\right)$. (These also correspond to the standard utilitarian approach in Allouch (2017)). For the star network with three consumers in Figure 4b, let us designate the central consumer as 1 and the two periphery consumers as 2 and 3. We can observe that $\mathbf{r}=(1, a, a)$ are Negishi weights.

\subsection{Cobb-Douglas preferences}

In the case of Cobb-Douglas preferences, $\left(u_{i}\left(x_{i}, Q_{i}\right)=x_{i}^{a} Q_{i}^{1-a}\right)$, we can further relate the feasibility of Pareto-improving reform to the network structure.

Corollary 3. Assume Cobb-Douglas preferences. There exist Negishi weights if and only if there exists $\mathbf{r} \in \mathbb{R}_{++}^{n}$ such that $\mathbf{R}=\mathbf{1}+$ ad, where $\mathbf{d}$ denotes degree.

Corollary 3 relates the existence of Negishi weights to the degree. It is easy to check for the existence of Negishi weights in two canonical network structures, regular and star. This is illustrated in Figure 4.

Corollary 4. Assume Cobb-Douglas preferences. If the component of contributors is regular or star, then there exists no Pareto improvement.

\section{Policy switch and the -1 eigenvalue}

Next we show how welfare targeting in networks, which accounts for heterogeneity of local interactions, provides a link between the neutrality and general welfare effects. Indeed, observe that when a Pareto-improving income redistribution exists, it holds that the 
donors' utility level must move in the opposite direction to the tax/subsidy scheme. In fact, this is an example of a weak transfer paradox. On the contrary, a strong transfer paradox corresponds to the case where the utility levels of both the donors and recipients move in the opposite direction to the tax/subsidy scheme - that is, those who give are better off and those who receive are worse off.

In order to further investigate welfare targeting in networks, we will focus our analysis on Cobb-Douglas preferences such that $u_{i}\left(x_{i}, Q_{i}\right)=x_{i}^{a} Q_{i}^{1-a}$.

Proposition 5. Assume Cobb-Douglas preferences. Given a component of contributors, if the small tax/subsidy scheme $\mathbf{s}$ is an eigenvector to the eigenvalue $\lambda$, then, for some $\kappa_{\lambda}>0$, it holds that

$$
\Delta \mathbf{v}(\mathbf{s})=\kappa_{\lambda}(\lambda+1) \mathbf{s}=\kappa_{\lambda} \mathbf{S}
$$

Proposition 5 shows that, in the case of Cobb-Douglas preferences, if the tax/subsidy scheme is an eigenvector, then its welfare impact is proportional to the corresponding neighbourhood scheme. Proposition 5 has several implications.

As shown in Corollary 1, the eigenvectors to the -1 eigenvalue characterise neutral tax/subsidy schemes. Observe that, the eigenvectors to the -1 either have no impact on consumption, if budget-balanced, or have an impact on consumption, if budgetunbalanced. In particular, a budget-unbalanced eigenvector to the -1 eigenvalue can be used to create tax revenue while keeping welfare unchanged, which can be redistributed for Pareto improvement, as shown in Proposition 4.

In addition, it holds that: $(i)$ if the eigenvalue is greater than -1 , then the utility levels of both the donors and the recipients move in the same direction as the tax/subsidy scheme, which corresponds to a 'normal' welfare impact and (ii) if the eigenvalue is smaller than -1 , then the utility levels of both the donors and the recipients move in the opposite direction to the tax/subsidy scheme, which corresponds to a 'paradoxical' welfare impact. Therefore, the welfare impact changes from one direction to the other depending on the eigenvalue, and the point at which the direction switches, -1 , is the point of policy neutrality.

In interpretation, the welfare impact can be thought of as a continuous policy function that changes sign, so at some point it must equal zero, which is the point of policy neutrality. 
The possibility of a paradoxical welfare effect may seem surprising. As shown in the utility maximisation, the change in utility of each consumer is an increasing function of his social wealth, $w_{i}+Q_{-i}$. So there will be two effects on a consumer's utility after income. As a direct effect, the endowment $w_{i}$ changes by $s_{i}$. There will also be an indirect effect on the consumer's social wealth because his neighbours' public good provision $Q_{-i}$ changes in the new equilibrium. Note that the direct effect always works in the direction of the normal or non-paradoxical welfare outcome and that a subtle change in the neighbours' public good provision is needed to generate the paradoxical welfare effect.

In the following we will show that the welfare effects can also be determined for a more general class of tax/subsidy schemes. Indeed, let $\mathcal{P}^{+}$(resp. $\mathcal{P}^{-}$) denote the linear space spanned by the eigenvectors to eigenvalues greater (resp. smaller) than -1 .

Proposition 6. Assume Cobb-Douglas preferences. Given a component of contributors and a small tax/subsidy scheme $\mathbf{s}$ :

1. If $\mathbf{s} \in \mathcal{P}^{+}$, then it holds that

$$
\Delta \mathbf{v}(\mathbf{s}) \cdot \mathbf{s}>0
$$

2. If $\mathbf{s} \in \mathcal{P}^{-}$, then it holds that

$$
\Delta \mathbf{v}(\mathbf{s}) \cdot \mathbf{s}<0
$$

Proposition 6 shows that a Law of welfare targeting holds in the case of Cobb-Douglas preferences. Indeed, it holds that if $\mathbf{s} \in \mathcal{P}^{+}$(resp. $\mathcal{P}^{-}$) then the utility levels of both the donors and the recipients move in the same (resp. opposite) direction as (resp. to) the tax/subsidy scheme, which corresponds to a normal (resp. paradoxical) welfare impact. Hence, the -1 eigenvalue is the point where the overall welfare effects change direction.

\section{Conclusion}

In this paper, we have shown that welfare targeting, which accounts for heterogeneity of local interactions, enables us to derive new results and policy insights. We have also identified the -1 eigenvalue of the network as the condition for neutral tax/subsidy schemes, a sufficient condition for Pareto improvement. and a policy switch point between consumers acting on or counteracting policy intervention. 


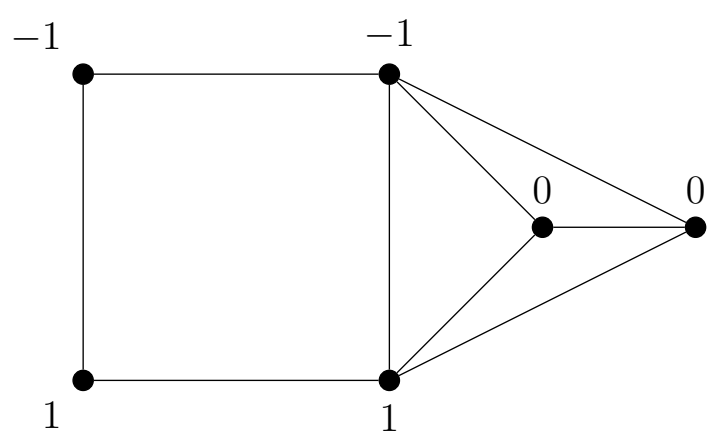

$\mathbf{S}_{3}$

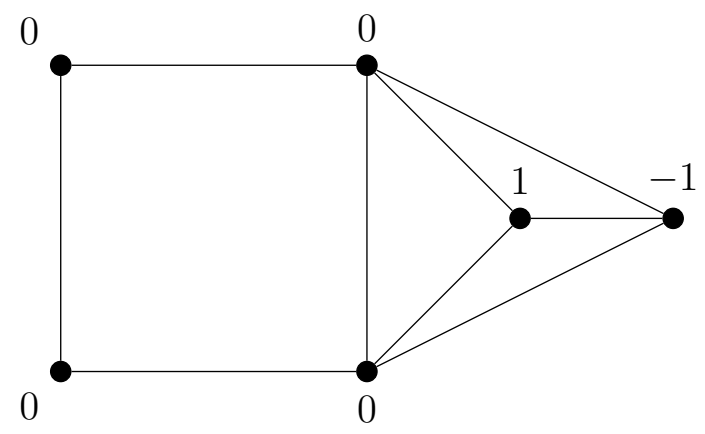

$\mathbf{S}_{4}$

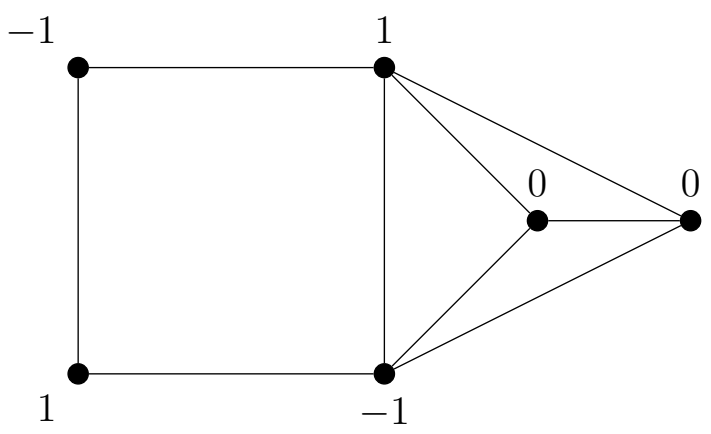

$\mathbf{s}_{6}$

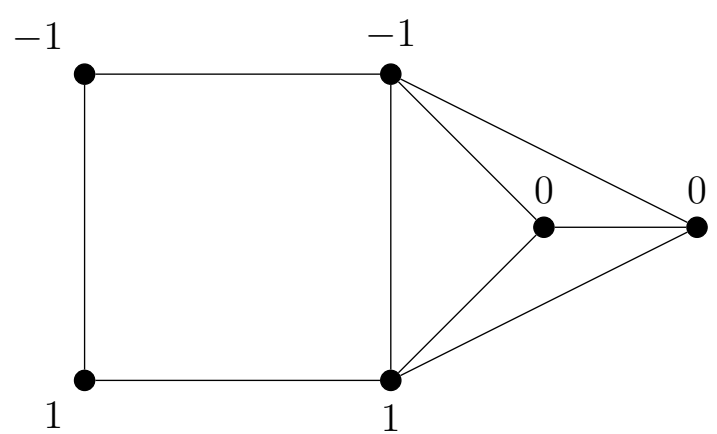

$\mathbf{S}_{3}$

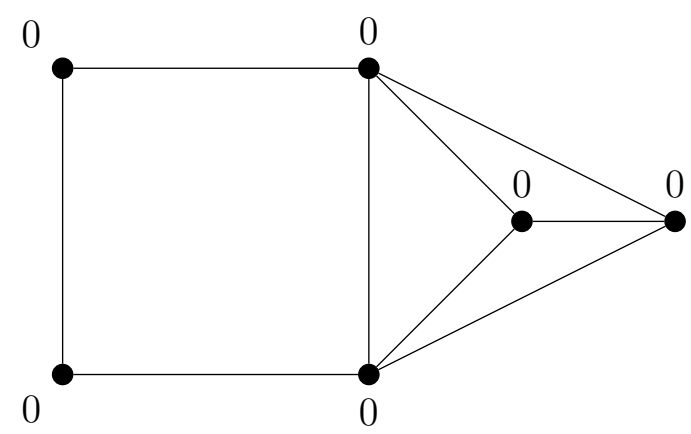

$\mathrm{S}_{4}$

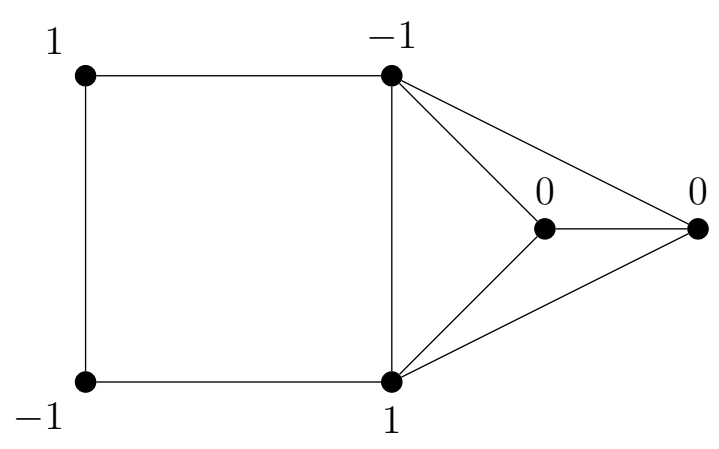

$\mathbf{S}_{6}$

Figure 5: A network with six consumers. Since the tax/subsidy schemes we will consider are also eigenvectors, in view of Proposition 5, we can measure their welfare impacts by the neighbourhood schemes. At the top, the welfare impact of $\mathbf{s}_{3}$ is normal, as $\left(\lambda_{3}=0\right)$. In the middle, the welfare impact of $\mathbf{s}_{4}$ is neutral, as $\lambda_{4}=-1$. At the bottom, the welfare impact of $\mathbf{s}_{6}$ is paradoxical, as $\lambda_{6}=-2$. 
The -1 eigenvalue is not a common measure of network analysis in any other fields, including sociology, computer science, and physics. ${ }^{5}$ Looking at tables of networks of at most five nodes in Cvetkovic, Rowlinson and Simic (1997) and of six nodes in Cvetkovic and Petric (1984), we notice that the -1 eigenvalue does occur frequently. More precisely, it occurs in more than half of the listed networks, sometimes with multiplicity. ${ }^{6}$ That said, even when the -1 eigenvalue is not present, its relative position in the spectrum is key to the policy switch and the law of welfare targeting. Given the policy relevance of the -1 eigenvalue, perhaps its presence and location in the spectrum could be usefully studied alongside other important network measures.

More generally, our findings for the private provision of public goods in networks highlight the interplay between strategic interactions of public goods in networks, whereby each agent's payoff depends on his own action and the sum of his neighbours' actions, and the underlying network structure. In this respect, we think our analysis could be useful for a variety of similar strategic interactions as in Bramoullé, Kranton and D'Amours (2014), Melo (2018), Parise and Ozdaglar (2018), and Galeotti, Golub and Goyal (2020). As a consequence, our findings can provide clear testable predictions for many applications including empirical and experimental work.

\section{A Appendix: Proofs}

Proof of Proposition 1 Given a tax/subsidy scheme s, adapting the proof of Proposition 1 in Allouch (2015) we have that

$$
\mathbf{q}^{\mathbf{s}}-\mathbf{q}^{*}=(\mathbf{I}+\mathbf{A G})^{-1}(\mathbf{I}-\mathbf{A}) \mathbf{s}
$$

\footnotetext{
${ }^{5}$ We are not aware of any mathematical or graph theory research on the relationship between the network structure and the -1 eigenvalue - with the exception of Aouchiche, Caporossi and Hansen (2013), Royle (2003), and Bıyıkoglu, Simic and Stanic (2011). All exceptions have a similar network structure to pure public goods. Nonetheless, as we have shown in several examples, the -1 eigenvalue can also have a significant welfare impact in a network structure that is very dissimilar from pure public goods.

${ }^{6}$ In a private communication, Sebastian Bervoets very kindly provided us with the remaining proportions with the -1 eigenvalue among networks of at most ten nodes, which are 0.504 for $N=7,0.44$ for $N=8,0.36$ for $N=9$, and 0.27 for $N=10$. While the ratios are clearly decreasing, they still remain significant, especially given the large number of networks.
} 
where $\mathbf{A}=\operatorname{diag}\left(1-\gamma_{i}^{\prime}\left(w_{i}+Q_{-i}^{*}\right)\right)_{i \in N}$. Since we normalised prices to $(1,1)$ and confined our analysis to a component of contributors, without loss of generality it holds that

$$
\nabla_{x} \mathbf{u}=\left(\frac{\partial u_{i}}{\partial x_{i}}\right)_{i \in N}=\nabla_{Q} \mathbf{u}=\left(\frac{\partial u_{i}}{\partial Q_{i}}\right)_{i \in N}=\mathbf{1}
$$

Next, we use $\mathbf{x}^{\mathbf{s}}-\mathbf{x}^{*}+\mathbf{q}^{\mathbf{s}}-\mathbf{q}^{*}=\mathbf{s}$ and Taylor approximation so that

$$
\begin{aligned}
\Delta \mathbf{v}(\mathbf{s}) & \approx \nabla_{x} \mathbf{u}\left(\mathbf{x}^{\mathbf{s}}-\mathbf{x}^{*}\right)+\nabla_{Q} \mathbf{u}\left(\mathbf{Q}^{\mathbf{s}}-\mathbf{Q}^{*}\right) \\
& =\mathbf{x}^{\mathbf{s}}-\mathbf{x}^{*}+\mathbf{Q}^{\mathbf{s}}-\mathbf{Q}^{*} \\
& =\mathbf{s}+\mathbf{Q}_{-i}^{\mathbf{s}}-\mathbf{Q}_{-i}^{*} \\
& =\mathbf{s}+\mathbf{G}\left(\mathbf{q}^{\mathbf{s}}-\mathbf{q}^{*}\right) \\
& =\mathbf{s}+\mathbf{G}(\mathbf{I}+\mathbf{A G})^{-1}(\mathbf{I}-\mathbf{A}) \mathbf{s} \\
& =\mathbf{s}+\mathbf{G}(\mathbf{I}+\mathbf{A G})^{-1}(\mathbf{I}+\mathbf{A G}-\mathbf{A}-\mathbf{A G}) \mathbf{s} \\
& =\mathbf{A}^{-1}(\mathbf{I}+\mathbf{A G})(\mathbf{I}+\mathbf{A G})^{-1} \mathbf{A}(\mathbf{I}+\mathbf{G}) \mathbf{s}-\mathbf{G}(\mathbf{I}+\mathbf{A G})^{-1} \mathbf{A}(\mathbf{I}+\mathbf{G}) \mathbf{s} \\
& =\mathbf{A}^{-1}(\mathbf{I}+\mathbf{A G})(\mathbf{I}+\mathbf{A G})^{-1} \mathbf{A}(\mathbf{I}+\mathbf{G}) \mathbf{s}-\mathbf{A}^{-1} \mathbf{A G}(\mathbf{I}+\mathbf{A G})^{-1} \mathbf{A}(\mathbf{I}+\mathbf{G}) \mathbf{s} \\
& =\mathbf{A}^{-1}(\mathbf{I}+\mathbf{A G})^{-1} \mathbf{A}(\mathbf{I}+\mathbf{G}) \mathbf{s} \\
& =\mathbf{A}^{-1}(\mathbf{I}+\mathbf{A G})^{-1} \mathbf{A S} .
\end{aligned}
$$

Proof of Corollary $\mathbf{1}$ Observe that a small tax/subsidy scheme $\mathbf{s}$ is neutral if and only if $\mathbf{S}=(\mathbf{I}+\mathbf{G}) \mathbf{s}=\mathbf{0}$, which is also equivalent to $\mathbf{s}$ being an eigenvector to the -1 eigenvalue.

Proof of Proposition 2 First, observe that

$$
\begin{aligned}
\mathbf{A}^{-1}(\mathbf{I}+\mathbf{A G})^{-1} \mathbf{A}(\mathbf{I}+\mathbf{G}) & =\mathbf{A}^{-1}(\mathbf{I}+\mathbf{A G})^{-1}(\mathbf{A}-\mathbf{I}+\mathbf{I}+\mathbf{A G}) \\
& =\mathbf{A}^{-1}(\mathbf{I}+\mathbf{A G})^{-1}(\mathbf{A}-\mathbf{I})+\mathbf{A}^{-1} \\
& =\mathbf{A}^{-1}(\mathbf{A}-\mathbf{I})^{-1}(\mathbf{A}-\mathbf{I})(\mathbf{I}+\mathbf{A G})^{-1}(\mathbf{A}-\mathbf{I}) \\
& +\mathbf{A}^{-1}(\mathbf{A}-\mathbf{I})^{-1}(\mathbf{I}+\mathbf{A G})(\mathbf{I}+\mathbf{A G})^{-1}(\mathbf{A}-\mathbf{I}) \\
& =\mathbf{A}^{-1}(\mathbf{A}-\mathbf{I})^{-1}[(\mathbf{A}-\mathbf{I})+(\mathbf{I}+\mathbf{A G})](\mathbf{I}+\mathbf{A G})^{-1}(\mathbf{A}-\mathbf{I}) \\
& =(\mathbf{A}-\mathbf{I})^{-1}(\mathbf{I}+\mathbf{G})(\mathbf{I}+\mathbf{A G})^{-1}(\mathbf{A}-\mathbf{I}) \\
& =(\mathbf{I}-\mathbf{A})^{-1}(\mathbf{I}+\mathbf{G})(\mathbf{I}+\mathbf{A G})^{-1}(\mathbf{I}-\mathbf{A}) .
\end{aligned}
$$

Therefore, there exist Negishi weights if and only if there exists $\mathbf{r}^{\prime} \in \mathbb{R}_{++}^{n}$ such that for any budget-balanced $\mathbf{s}$ it holds that

$$
\mathbf{r}^{\prime} \cdot \Delta \mathbf{v}(\mathbf{s}) \approx \mathbf{r}^{\prime}(\mathbf{I}-\mathbf{A})^{-1}(\mathbf{I}+\mathbf{G})(\mathbf{I}+\mathbf{A G})^{-1}(\mathbf{I}-\mathbf{A}) \mathbf{s}=0
$$


which is equivalent to there exists $\alpha>0$ such that

$$
\mathbf{r}^{\prime}(\mathbf{I}-\mathbf{A})^{-1}(\mathbf{I}+\mathbf{G})(\mathbf{I}+\mathbf{A G})^{-1}(\mathbf{I}-\mathbf{A})=\alpha \mathbf{1}
$$

Let

$$
\mathbf{r}=\frac{1}{\alpha} \mathbf{r}^{\prime}(\mathbf{I}-\mathbf{A})^{-1}
$$

Then, there exist Negishi weights if and only if

$$
\mathbf{r}(\mathbf{I}+\mathbf{G})(\mathbf{I}+\mathbf{A G})^{-1}(\mathbf{I}-\mathbf{A})=\mathbf{1}
$$

which is equivalent to

$$
\mathbf{R}=\mathbf{1}(\mathbf{I}-\mathbf{A})^{-1}(\mathbf{I}+\mathbf{A G})
$$

Proof of Proposition 3 Let $\mathcal{V}$ denote the linear subspace spanned by utility changes from budget-balanced tax/subsidy schemes:

$$
\mathcal{V}=\left\{\Delta \mathbf{v}(\mathbf{s}) \in \mathbb{R}^{n} \mid \Delta \mathbf{v}(\mathbf{s})=\mathbf{A}^{-1}(\mathbf{I}+\mathbf{A G})^{-1} \mathbf{A}(\mathbf{I}+\mathbf{G}) \mathbf{s} \text { for a budget-balanced } \mathbf{s}\right\} .
$$

Therefore, it follows from Corollary $3^{\prime}$ of Ben-Israel (1964) that there are only two (mutually exclusive) possibilities, $(a)$ and $(b)$ :

$$
\begin{aligned}
& \text { (a) } \mathcal{V} \cap \mathbb{R}_{+}^{n} \neq\{0\} \Longleftrightarrow \mathcal{V}^{\perp} \cap \mathbb{R}_{++}^{n}=\varnothing . \\
& \text { (b) } \mathcal{V} \cap \mathbb{R}_{+}^{n}=\{0\} \Longleftrightarrow \mathcal{V}^{\perp} \cap \mathbb{R}_{++}^{n} \neq \varnothing .
\end{aligned}
$$

If $(a)$ then there exists a Pareto-improving income redistribution in the subspace $\mathcal{V}$.

If (b) then $\mathcal{V}^{\perp}$ contains $\mathbf{r}^{\prime} \in \mathbb{R}_{++}^{n}$ such that $\mathbf{r}^{\prime} \cdot \Delta \mathbf{v}(\mathbf{s})=0$ for any budget-balanced $\mathbf{s}$, which is equivalent to the existence of Negishi weights.

Proof of Proposition 4 Suppose there exists no Pareto-improving income redistribution. Then there exist Negishi weights, or equivalently there exist $\mathbf{r} \in \mathbb{R}_{++}^{n}$ such that

$$
\mathbf{R}=\mathbf{1}(\mathbf{I}-\mathbf{A})^{-1}(\mathbf{I}+\mathbf{A G}),
$$

or equivalently,

$$
\mathbf{R}(\mathbf{I}+\mathbf{A G})^{-1}(\mathbf{I}-\mathbf{A})=\mathbf{1}
$$

Since -1 is a main eigenvalue, there exists an eigenvector $\mathbf{s}$ to the -1 eigenvalue such that $\mathbf{1} \cdot \mathbf{s} \neq 0$. Then it holds that

$$
0=\mathbf{r} \cdot \mathbf{S}(\mathbf{I}+\mathbf{A G})^{-1}(\mathbf{I}-\mathbf{A})=\mathbf{s} \cdot \mathbf{R}(\mathbf{I}+\mathbf{A G})^{-1}(\mathbf{I}-\mathbf{A})=\mathbf{s} \cdot \mathbf{1} \neq 0 .
$$

which is a contradiction. 
Proof of Corollary 2 Note that, in an equal neighbourhood component of contributors, the vector with all 1 entries in one group and all -1 entries in the other group is an eigenvector to the -1 eigenvalue. This eigenvector to the -1 eigenvalue is also budgetunbalanced since the two groups have unequal sizes. Hence, -1 is a main eigenvalue. The existence of Pareto-improving income redistribution follows from Proposition 4.

Proof of Corollary 3 Given Cobb-Douglas preferences, $u_{i}\left(x_{i}, Q_{i}\right)=x_{i}^{a} Q_{i}^{1-a}$, for each consumer $i=1, \ldots, n$, then it holds that $\gamma_{i}^{\prime}(\cdot)=1-a$. Hence $\mathbf{A}=a \mathbf{I}$. It follows from Proposition 2 there exist Negishi weights if and only if there exists $\mathbf{r}^{*} \in \mathbb{R}_{++}^{n}$ such that

$$
\mathbf{R}^{*}=\frac{1}{1-a} \mathbf{1}(\mathbf{I}+a \mathbf{G})=\frac{1}{1-a}(\mathbf{1}+a \mathbf{d})
$$

Let $\mathbf{r}=(1-a) \mathbf{r}^{*}$. Then, there exist Negishi weights if and only if there exists $\mathbf{r} \in \mathbb{R}_{++}^{n}$ such that

$$
\mathbf{R}=\mathbf{1}+a \mathbf{d}
$$

Proof of Corollary 4 For a regular network, consider $\mathbf{r}=\frac{1+a d}{1+d} \mathbf{1}$, where $d$ is each consumer's degree. For a star network, consider $\mathbf{r}$ such that $r_{c}=1$ for the central consumer and $r_{p}=a$ for the periphery consumers.

Proof of Proposition 5 From the proof of Proposition 2, it follows that

$$
\Delta \mathbf{v}(\mathbf{s})=(\mathbf{I}+a \mathbf{G})^{-1}(\mathbf{I}+\mathbf{G}) \mathbf{s} .
$$

Therefore, if the the tax/subsidy scheme $\mathbf{s}$ is an eigenvector to the eigenvalue $\lambda$, then it holds that

$$
\Delta \mathbf{v}(\mathbf{s})=\kappa_{\lambda}(\lambda+1) \mathbf{s}=\kappa_{\lambda} \mathbf{S},
$$

where $\kappa_{\lambda}=\frac{1}{1+a \lambda}$. Having assumed network normality, we note that this is equivalent to $a \in] 0,-\frac{1}{\lambda_{\min }(\mathbf{G})}\left[\right.$ in the Cobb-Douglas preferences case, which ensures that $\kappa_{\lambda}$ is positive.

Proof of Proposition 6 Let $\mathbf{s} \in \mathcal{P}^{+}\left(\right.$resp. $\left.\mathbf{s} \in \mathcal{P}^{-}\right)$. Then there exist $\left(\mathbf{s}_{k}\right)_{k \in K}$ that are eigenvectors to eigenvalues $\left(\lambda_{k}\right)_{k \in K}$ such that $\lambda_{k}>-1$ (resp. $\lambda_{k}<-1$ ) and real numbers $\left(\mu_{k}\right)_{k \in K}$ such that $\mathbf{s}=\sum_{k \in K} \mu_{k} \mathbf{s}_{k}$. From Proposition 5, it follows that

$$
\Delta \mathbf{v}(\mathbf{s})=\Delta \mathbf{v}\left(\sum_{k \in K} \mu_{k} \mathbf{s}_{k}\right)=\sum_{k \in K} \mu_{k} \Delta \mathbf{v}\left(\mathbf{s}_{k}\right)=\sum_{k \in K} \mu_{k} \kappa_{\lambda_{k}}\left(\lambda_{k}+1\right) \mathbf{s}_{k} .
$$

Therefore, it holds that

$$
\Delta \mathbf{v}(\mathbf{s}) \cdot \mathbf{s}=\left(\sum_{k \in K} \mu_{k} \kappa_{\lambda_{k}}\left(\lambda_{k}+1\right) \mathbf{s}_{k}\right) \cdot\left(\sum_{k \in K} \mu_{k} \mathbf{s}_{k}\right)=\sum_{k \in K} \mu_{k}^{2} \kappa_{\lambda_{k}}\left(\lambda_{k}+1\right)>0(\text { resp. }<0) .
$$




\section{References}

Abbott, Joshua K. and Eli P. Fenichel (2014) "Following the golden rule: Negishi welfare weights without apology," Unpublished manuscript.

Acemoglu, Daron, Azarakhsh Malekian, and Asu Ozdaglar (2016) "Network security and contagion," Journal of Economic Theory, Vol. 166, pp. 536-585.

Ahmad, Ehtisham and Nicholas Stern (1984) "The theory of reform and Indian indirect taxes," Journal of Public Economics, Vol. 25, pp. 259-298.

Akbarpour, Mohammad, Suraj Malladi, and Amin Saberi (2020) "Just a Few Seeds More: Value of Network Information for Diffusion," SSRN Scholarly Paper ID 3062830, Social Science Research Network, Rochester, NY.

Allouch, Nizar (2015) "On the private provision of public goods on networks," Journal of Economic Theory, Vol. 157, pp. 527-552.

- (2017) "The cost of segregation in (social) networks," Games and Economic Behavior, Vol. 106, pp. 329-342.

Allouch, Nizar and Maia King (2019) "Constrained public goods in networks," Journal of Public Economic Theory, Vol. 21, pp. 895-902.

Aouchiche, Mustapha, Gilles Caporossi, and Pierre Hansen (2013) "Open problems on graph eigenvalues studied with AutoGraphiX," EURO Journal on Computational Optimization, Vol. 1, pp. 181-199.

Becker, Gary S. (1974) "A theory of social interactions," Working Paper 42, National Bureau of Economic Research.

Belhaj, Mohamed and Frédéric Deroïan (2019) "Group targeting under networked synergies," Games and Economic Behavior, Vol. 118, pp. 29-46.

Ben-Israel, Adi (1964) "Notes on linear inequalities, I: The intersection of the nonnegative orthant with complementary orthogonal subspaces," Journal of Mathematical Analysis and Applications, Vol. 9, pp. 303-314.

Bergstrom, Ted, Lawrence Blume, and Hal Varian (1986) "On the private provision of public goods," Journal of Public Economics, Vol. 29, pp. 25-49. 
Bourlès, Renaud, Yann Bramoullé, and Eduardo Perez-Richet (2017) "Altruism in networks," Econometrica, Vol. 85, pp. 675-689.

Bramoullé, Yann and Rachel Kranton (2007) "Public goods in networks," Journal of Economic Theory, Vol. 135, pp. 478-494.

Bramoullé, Yann, Rachel Kranton, and Martin D'Amours (2014) "Strategic interaction and networks," American Economic Review, Vol. 104, pp. 898-930.

Bıyıkoglu, Türker, Slobodan K. Simic, and Zoran Stanic (2011) "Some notes on spectra of cographs," Ars Combin, Vol. 100, pp. 421-434.

Chen, Ying-Ju, Yves Zenou, and Junjie Zhou (2018) "Multiple activities in networks," American Economic Journal: Microeconomics, Vol. 10, pp. 34-85.

Cvetkovic, Dragos (1970) "The generating function for variations with restrictions and paths of the graph and self-complementary graphs," Univ. Beograd, Publ. Elektrotehn. Fak. Ser. Mat., pp. 27-34.

Cvetkovic, Dragos and Milenko Petric (1984) "A table of connected graphs on six vertices," Discrete Mathematics, Vol. 50, pp. 37-49.

Cvetkovic, Dragos, Peter Rowlinson, and Slobodan Simic (1997) Eigenspaces of graphs, Cambridge ; New York: Cambridge University Press.

Dixit, Avinash (1975) "Welfare effects of tax and price changes," Journal of Public Economics, Vol. 4, pp. 103-123.

Elliott, Matthew and Benjamin Golub (2019) "A network approach to public goods," Journal of Political Economy, Vol. 127, pp. 730-776.

Galeotti, Andrea, Benjamin Golub, and Sanjeev Goyal (2020) "Targeting Interventions in Networks," Econometrica, Vol. 88, pp. 2445-2471.

Galeotti, Andrea and Sanjeev Goyal (2010) "The law of the few," American Economic Review, Vol. 100, pp. 1468-1492.

Galeotti, Andrea, Sanjeev Goyal, Matthew O. Jackson, Fernando Vega-Redondo, and Leeat Yariv (2010) "Network games," Review of Economic Studies, Vol. 77, pp. 218244. 
Ghiglino, Christian and Sanjeev Goyal (2010) "Keeping up with the neighbors: social interaction in a market economy," Journal of the European Economic Association, Vol. 8, pp. 90-119.

Guesnerie, Roger (1977) "On the direction of tax reform," Journal of Public Economics, Vol. 7, pp. 179-202.

Kinateder, Markus and Luca Paolo Merlino (2017) "Public goods in endogenous networks," American Economic Journal: Microeconomics, Vol. 9, pp. 187-212.

Li, Jian, Junjie Zhou, and Ying-Ju Chen (2021) "The Limit of Targeting in Networks," SSRN Scholarly Paper ID 3776944, Social Science Research Network, Rochester, NY.

López-Pintado, Dunia (2017) "Influence networks and public goods," SERIEs, Vol. 8, pp. $97-112$.

Melo, Emerson (2018) "A variational approach to network games," SSRN Scholarly Paper ID 3143468, Social Science Research Network, Rochester, NY.

Negishi, Takashi (1960) "Welfare economics and existence of an equilibrium for a competitive economy," Metroeconomica, Vol. 12, pp. 92-97.

Parise, Francesca and Asuman Ozdaglar (2018) "Graphon games," arXiv:1802.00080 [cs], arXiv: 1802.00080 .

Royle, Gordon F. (2003) "The rank of a cograph," the electronic journal of combinatorics, pp. N11-N11.

Stanton, Elizabeth A. (2011) "Negishi welfare weights in integrated assessment models: the mathematics of global inequality," Climatic Change, Vol. 107, pp. 417-432.

Sun, Yang, Wei Zhao, and Junjie Zhou (2021) "Structural Interventions in Networks," arXiv preprint arXiv:2101.12420.

Ushchev, Philip and Yves Zenou (2020) "Social norms in networks," Journal of Economic Theory, Vol. 185, p. 104969.

Yang, Zili and William D. Nordhaus (2006) "Magnitude and direction of technological transfers for mitigating GHG emissions," Energy Economics, Vol. 28, pp. 730-741. 